\title{
TRATAMENTO DOS RESÍDUOS DE GESSO DA CONSTRUÇÃO E DA DEMOLIÇÃO-RCD PARA A PRODUÇÃO DE GESSO BETA RECICLADO
}

\author{
H. J. B. de LIMA FILHO ${ }^{1}$, J. C. S. LIMA ${ }^{2}$,M. BENACHOUR ${ }^{2}$, V. A. dos SANTOS ${ }^{1}$ \\ ${ }^{1}$ Universidade Católica de Pernambuco, Centro de Ciências e Tecnologia (CCT) \\ ${ }^{2}$ Universidade Federal de Pernambuco, Departamento de Engenharia Química (DEQ) \\ E-mail para contato: hilario@unicap.br
}

\begin{abstract}
RESUMO - O presente trabalho de pesquisa teve como objetivo principal determinar as condições ideais de operação do forno rotativo piloto para calcinar os resíduos de bloco de gesso (RBG), e produzir um gesso beta reciclado com propriedades físico-químicas e mecânicas conforme as especificações das normas vigentes brasileiras (NBR's), propondo uma alternativa economicamente interessante para os geradores deste resíduo. Aplicando o Delineamento Composto Central Rotacional (DCCR) como metodologia, foram avaliados três fatores: velocidade de rotação, temperatura de controle e vazão mássica de sólidos. Uma vez identificada às condições ótimas de calcinação, foram realizados experimentos com adição de gipsita aos RBG com seguintes frações mássicas: 0 (resíduo puro), 50, 70, 80 e 88\%. O processo de calcinação dos RBG apresentou resultados promissores, produzindo um gesso beta reciclado que cumpre parcialmente as exigências normativas, utilizando uma fração igual ou maior do que $80 \%$ de gipsita.
\end{abstract}

\section{INTRODUÇÃO}

$\mathrm{O}$ mineral gipsita é o sulfato de cálcio dihidratado $\left(\mathrm{CaSO}_{4} 2 \mathrm{H}_{2} \mathrm{O}\right)$, que pode ser encontrado em diversas regiões do mundo apresentando um amplo e diversificado campo de utilizações. $\mathrm{O}$ grande interesse pela gipsita é atribuído a uma característica peculiar que consiste na facilidade de desidratação e posterior reidratação. Durante o processo de calcinação a gipsita perde $3 / 4$ da água de cristalização, convertendo-se a um sulfato de cálcio hemihidratado $\left(\mathrm{CaSO}_{4} 1 / 2 \mathrm{H}_{2} \mathrm{O}\right)$, e quando misturado com água pode ser modelado e trabalhado antes de endurecer e adquirir a consistência mecânica da forma rehidratada e estável (BALTAR et al., 2008).

O beneficiamento da gipsita para a produção de gesso envolve as seguintes operações: (1) catação manual; (2) britagem; (3) rebritagem; (4) peneiramento (usado em apenas algumas empresas); (5) ensilamento; (6) calcinação; (7) estabilização térmica em silos; (8) moagem (de acordo com a especificação de mercado para o produto); (9) ensilamento e (10) ensacamento. O produto resultante das operações de cominuição deve apresentar uma distribuição granulométrica uniforme a fim de possibilitar uma desidratação por igual para as partículas de gipsita (BALTAR et al., 2004). Dependendo do processo de calcinação da gipsita obtêm-se variedades de hemihidrato conhecidas 


\section{9 a 22 de outubro de 2014 \\ Florianópolis/SC}

como gesso beta e gesso alfa. Nos fornos que proporcionam uma calcinação sob pressão atmosférica, produz-se o gesso beta, enquanto que nos fornos do tipo autoclave, serão utilizados na produção do gesso alfa. $\mathrm{O}$ gesso beta é utilizado na indústria da construção civil, cimenteira e de modelagem, enquanto que o gesso alfa apresenta uma menor demanda de água para a formação da pasta, resultando em produtos de melhor qualidade, com aplicações na indústria cerâmica (moldes), na medicina (gesso ortopédico) e na odontologia (produção de próteses dentárias).

A preservação ambiental e uma preocupação mundial. Na construção civil não é diferente. Apesar de seus reconhecidos impactos socioeconômicos para o país, como alta geração de empregos, renda, viabilização de moradias, infra-estrutura, estradas e outros, ela ainda carece de uma firme política para a destinação de seus resíduos sólidos, principalmente nos grandes centros urbanos (SINDUSCON-MG, 2005), uma vez que grande parte destes resíduos é depositada de forma irregular, como lixões clandestinos, margens de rios e córregos, ou em terrenos baldios, sendo um dos principais responsáveis pelo esgotamento de áreas de aterros em cidades de médio e grande porte. (SILVA et al. 2006)

Nesse contexto, o presente trabalho teve como objetivo principal determinar as condições ótimas de operação do forno rotativo piloto para calcinação dos resíduos de bloco de gesso, produzindo um gesso beta reciclado com propriedades físico-químicas e mecânicas conforme as especificações das normas vigentes brasileiras (NBR's), procurando propor uma alternativa viável para os geradores destes resíduos.

\section{METODOLOGIA}

\subsection{Forno Piloto de Cilindro Rotativo}

No Laboratório de Processos Catalíticos da Universidade Federal de Pernambuco encontra-se um forno rotativo para a calcinação parcial do minério de gesso. $\mathrm{O}$ forno possui um diâmetro interno de $0,9 \mathrm{~m}$ e um comprimento total de 5,46m como mostra a Figura 1 . É confeccionado em chapa de aço carbono de $0.006 \mathrm{~m}$ de espessura e possui internamente quatro chicanas axiais, e uniformemente distribuídas. Duas destas chicanas, diametralmente opostas, possuem terminais com inclinação de 90 graus, para promoverem um preenchimento mais uniforme possível da seção transversal do forno com os sólidos; provocando um maior contato entre os componentes da mistura sólido- gases de calcinação. Esta mistura sólido-gases é do tipo concorrente.

A alimentação de sólidos para o sistema é realizada com auxílio de um elevador de caçambas que faz a descarga em um silo. Este possui em sua base uma válvula rotativa que controla a vazão de sólidos, que descem para a câmara de combustão (também situada abaixo do silo) com auxilio de uma calha de formato retangular. Os sólidos são conduzidos à saída do forno com auxilio da inclinação do mesmo. O gesso produzido é então descarregado em um recipiente de coleta. Os gases quentes que circulam pelo forno são originados da combustão do gás natural e são basicamente compostos de gás natural não reagido bem como produtos de sua combustão, do ar atmosférico e vapor de água proveniente da desidratação do minério gipsita. 
Para melhor confiabilidade de resultados foi considerado que os experimentos tiveram início somente quando o forno rotativo piloto entrou em regime permanente. Foi, então, avaliada a contribuição dos três parâmetros operacionais que têm influência sobre o grau de hidratação: fluxo contínuo de alimentação (Qs), a temperatura da zona de pré - calcinação (Tc) e da velocidade de rotação do forno rotativo piloto (n).

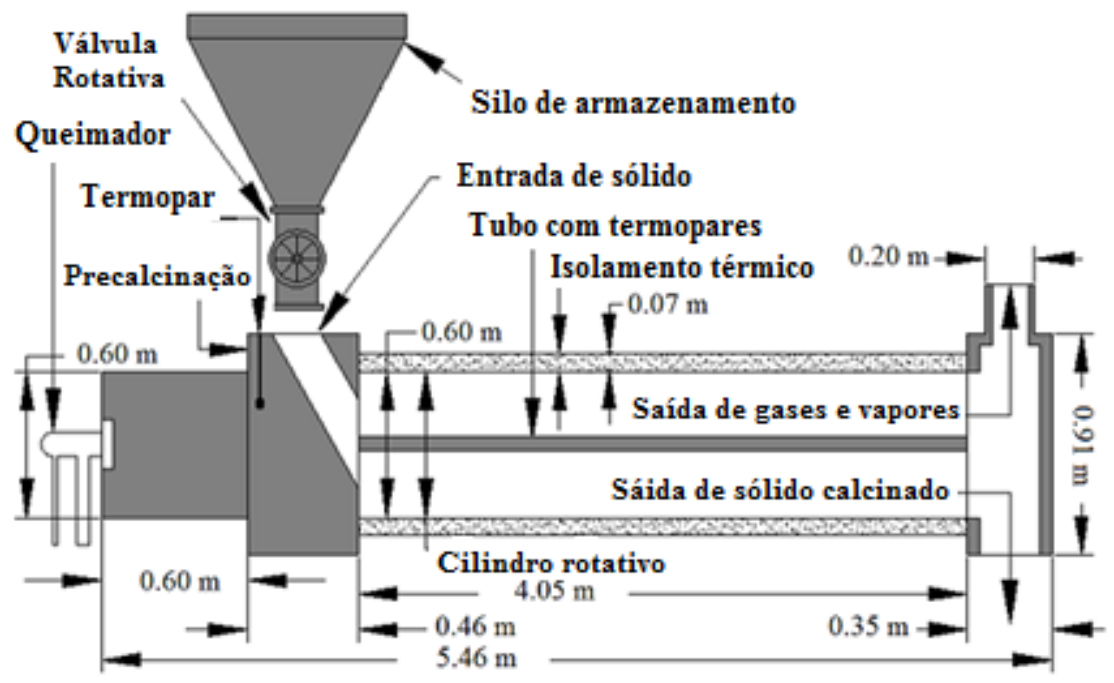

Figura 1 - Vista lateral esquemática do piloto forno rotativo.

\subsection{Planejamento Fatorial Experimental}

A principal vantagem do planejamento de experimentos foi efetuar uma análise dos parâmetros (supracitados) que possuíssem maiores influencia na variação do teor de hidratação (\%) da gipsita calcinada no forno rotativo piloto, e com base nesta análise obter uma equação de modelo que represente de forma confiável as condições ótimas de calcinação da gipsita para a produção de gesso beta. A partir disto estes parâmetros foram utilizados para criar um planejamento fatorial completo, com três níveis e três fatores, como está disposto na Tabela 1.

Tabela 1 - Valores e níveis utilizados no planejamento fatorial

\begin{tabular}{|c|c|c|c|}
\hline \multirow{2}{*}{$\begin{array}{l}\text { Variáveis independentes } \\
\text { (variáveis controladas) }\end{array}$} & \multicolumn{3}{|c|}{ Níveis } \\
\hline & $\begin{array}{c}-1 \\
\text { (baixo) }\end{array}$ & $\begin{array}{c}0 \\
\text { (médio) }\end{array}$ & $\begin{array}{c}1 \\
\text { (alto) }\end{array}$ \\
\hline Temperatura de controle da combustão $\left({ }^{\circ} \mathrm{C}\right)$ & 450 & 500 & 550 \\
\hline Velocidade de rotação do cilindro (rpm) & 1 & 2 & 3 \\
\hline Vazão de alimentação de sólidos (Kg/h) & 105 & 175 & 260 \\
\hline
\end{tabular}

\subsection{Caracterização do Gesso}


As amostras do sólido calcinado foram caracterizadas de acordo com as normas vigentes (NBR 12127, NBR 12128, NBR 12129 e NBR 12130), e suas propriedades físico-químicas e mecânicas foram quantificadas e comparadas com as especificações exigidas pela norma NBR 13207 para o recebimento do gesso a ser utilizado para fundição ou revestimento. Estão dispostos a seguir os testes sugeridos pelas NBR's e suas respectivas faixas de aceitação:

- Teor de hidratação - entre 4,2 e $6,2 \%$

- Massa Unitária - limite inferior maior que $700 \mathrm{~kg} / \mathrm{m}^{3}$;

- Tempo de pega - inicial: 4-10 min, final: 20 - 45min;

- Resistencia mecânica - acima de 8,4MPa;

\section{RESULTADOS E DISCUSSÃO}

\subsection{Resposta do planejamento dando o ponto ótimo}

As Figuras 2, 3 e 4 ilustram as superfícies de resposta e curvas de contorno para as variáveis dependentes em função do grau de hidratação. Um aumento nos resultados de Tc numa redução do grau de hidratação do seguinte calcinação sólido é observado na Figura 2. Este fenômeno que diz respeito à transferência de calor para as partículas de dihidrato de sulfato de cálcio no forno por condução, convecção e radiação. Um aumento na Qs leva a uma maior quantidade de material que entra no forno, provocando uma redução da temperatura do sólido no ponto de saída, resultando em uma baixa percentagem de conversão. A análise de superfície de resposta revela uma região ótima para o grau de hidratação entre 6,20 e $6,21 \%$, ajustando o processo com os seguintes valores dos parâmetros de operação: Qs $=175 \mathrm{~kg} / \mathrm{h}, \mathrm{Tc}=500^{\circ} \mathrm{C}$ e $\mathrm{n}=2 \mathrm{rpm}$.

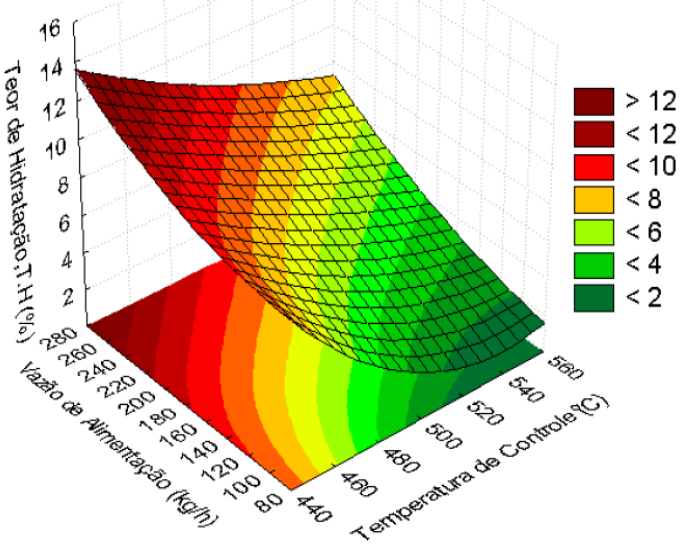

a) superfície de resposta.

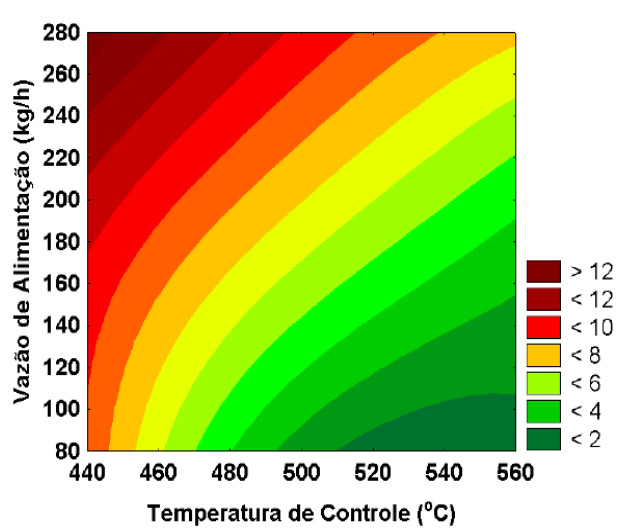

b) plano.

Figura 2 - a)Superfície de resposta e b)Plano para o Teor de Hidratação em função da Temperatura de Controle versus Vazão de Alimentação de Sólidos. 


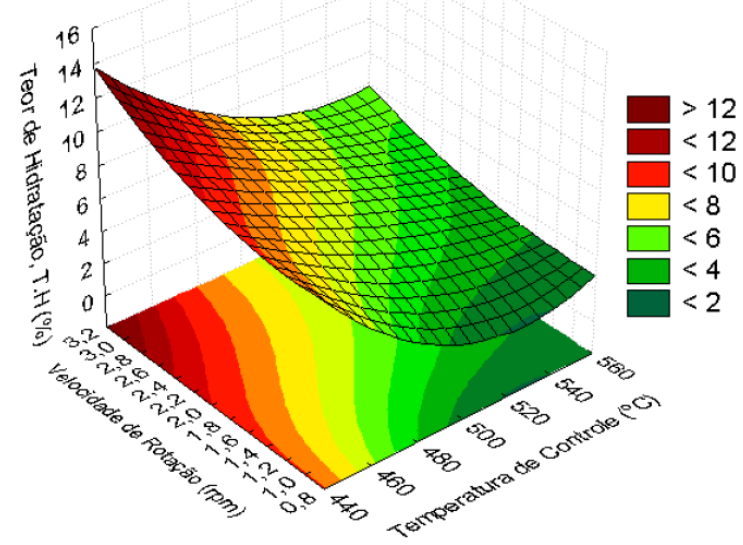

a) superfície de resposta.

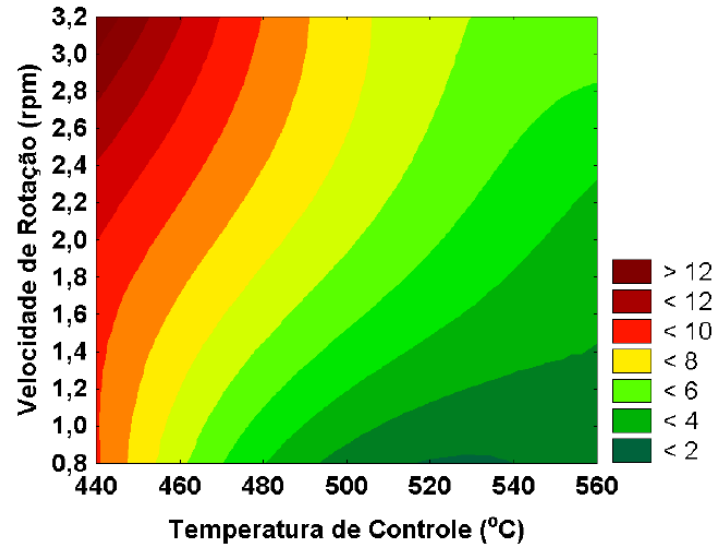

b) plano.

Figura 3 - a)Superfície de resposta e b)Plano para o teor de Hidratação em função da temperatura de controle versus velocidade de rotação do cilindro.

A Figura 3 mostra a variação de temperatura (Tc), em função da velocidade de rotação (n) com um fluxo de alimentação sólida constante. Um aumento na velocidade de rotação implica valores mais elevados em relação ao grau de hidratação, como velocidades mais rápidas reduzir o tempo de permanência do material, o que também diminui a permuta de calor no forno e produz um sulfato de cálcio semi-hidratado, com um menor grau de conversão.

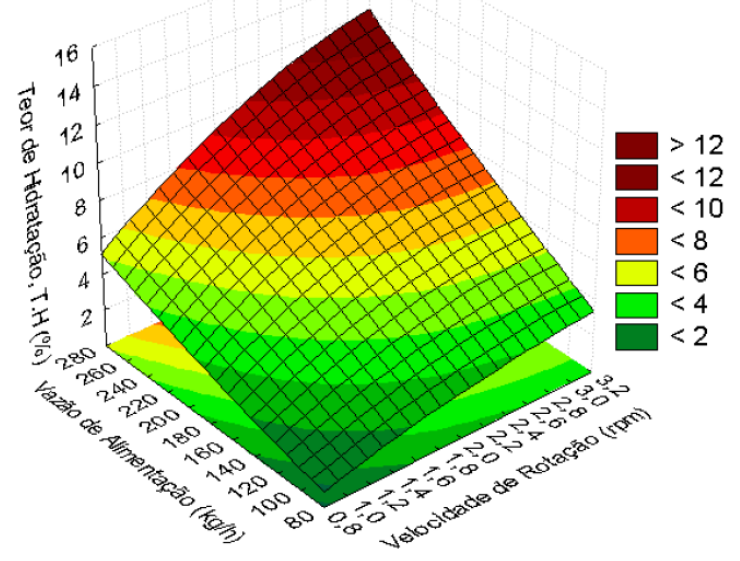

a) superfície de resposta.

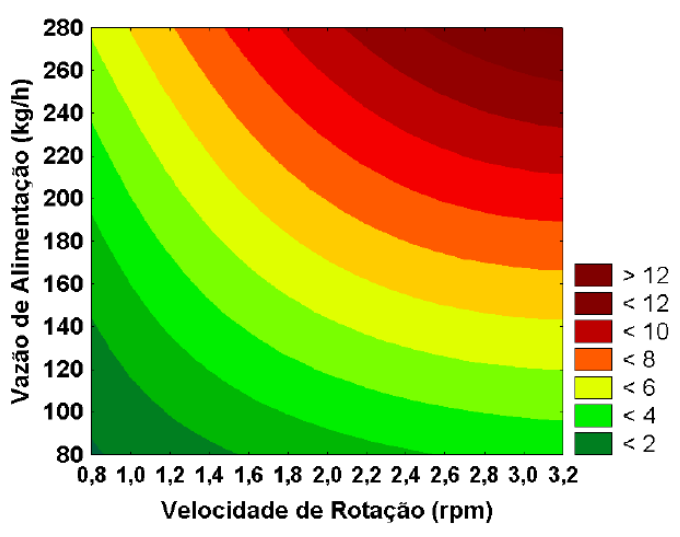

b) plano.

Figura 4 - a) Superfície de respostas e b) curvas de contorno para o Teor de Hidratação em função da Velocidade de Rotação do Cilindro versus Vazão de alimentação.

Analisando a figura 4, com a temperatura definida, os maiores valores do grau de hidratação são alcançados quando, simultaneamente, aumentando a velocidade de rotação e o fluxo de alimentação 
de sólidos. Da mesma forma, os menores valores da variável resposta são encontradas quando n e Qs são ajustados aos seus valores mais baixos.

\subsection{Caracterização do Gesso}

Com base nas condições otimizadas, a calcinação do resíduo de construção e demolição (RCD) foi realizada sem variações nos fatores controlados, os quais foram fixados em um fluxo de alimentação de sólido de $175 \mathrm{~kg} / \mathrm{h}$, temperatura de combustão de $500{ }^{\circ} \mathrm{C}$ e velocidade de rotação de $2 \mathrm{rpm}$. Todos RCD foram devidamente peneirados através de uma peneira de malha de $2 \mathrm{~mm}$ antes de ser colocado no forno. Os ensaios foram realizados através da mistura de gesso com o RCD no ponto de alimentação, com as seguintes proporções de minério de gesso: 0, 50, 70, 80 e $88 \%$.

O hemi-hidrato de sulfato de cálcio (gesso beta) reciclado produzido pela calcinação de RCD e gesso nas proporções acima mencionadas foi submetido a ensaios para a caracterização das propriedades físicas e mecânicas (Figuras 5 e 6). Estas propriedades (resistência mecânica, tempo de configuração inicial e hora configuração final) foram determinados utilizando amostras de ensaios que consiste reciclado sulfato de cálcio hemi-hidratado beta (gesso), com água a proporção de gesso de 0,7 (LAMBE e OFFUTT, 1954). As outras propriedades foram determinadas usando o próprio gesso reciclado.

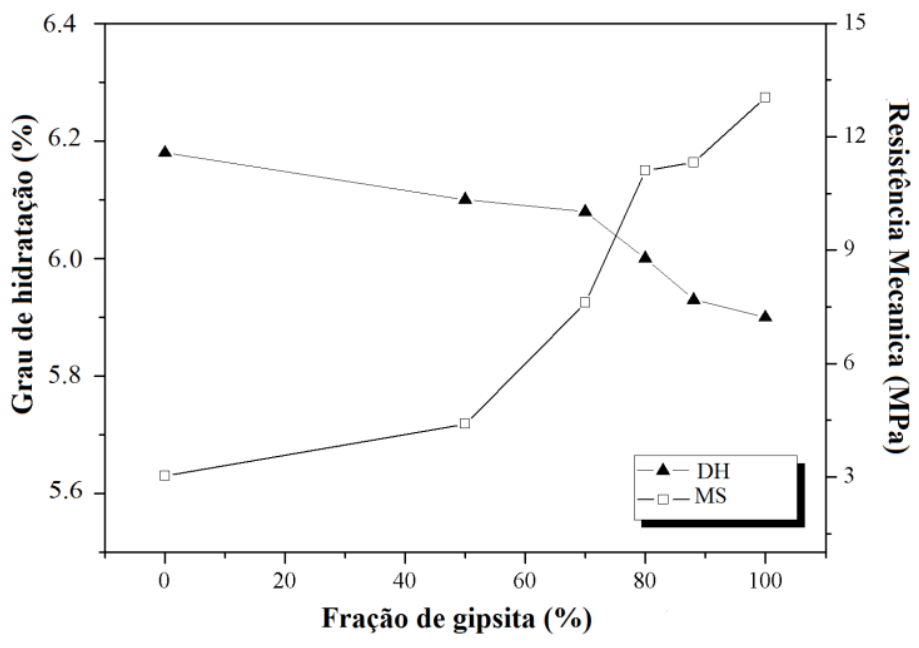

Figura 5 - Grau de hidratação e resistência à compressão em função da fração de gesso (RCD,

$$
\mathrm{Qs}=175 \mathrm{~kg} / \mathrm{h} \text { e } \mathrm{n}=2 \mathrm{rpm})
$$

Analisando a Figura 5, o grau de hidratação do semi-hidrato de sulfato de cálcio diminui após a adição de quantidades maiores de gesso no processo de calcinação. A variação considerável na umidade do RCD é o principal fator que explica este fenômeno, como o gesso usado o presente estudo tem baixo teor de umidade $(0,50 \%)$. A variação da resistência mecânica em função da proporção de gesso adicionada também é evidente, tal como um aumento na quantidade de gesso na mistura levou a um aumento na resistência mecânica. A Norma Brasileira 13207 estipula que o gesso beta deve ter resistência à compressão mínima de 8,40 MPa. Este requisito foi satisfeito quando o 
processo de calcinação operado com proporções de gesso igual ou superior a $80 \%$. Devido ao fraco desempenho do RCD puro calcinado (sem gipsita), é possível que a hidratação do gesso reciclado produza uma estrutura com má formação cristalina (alto grau de porosidade), afetando negativamente a resistência mecânica.

O tempo de secagem final é o tempo decorrido desde o momento em que o hemi-hidrato de sulfato de cálcio entra em contato com a água até que a superfície da pasta endureça completamente (SINGH e MIDDENDORF, 2007). Analisando a Figura 6, o ajuste final de tempo aumenta com a adição de gipsita e a menor fração da RCD produz um tempo de ajuste final a menos de 20 minutos, o que é uma exigência da norma brasileira NBR 13207.

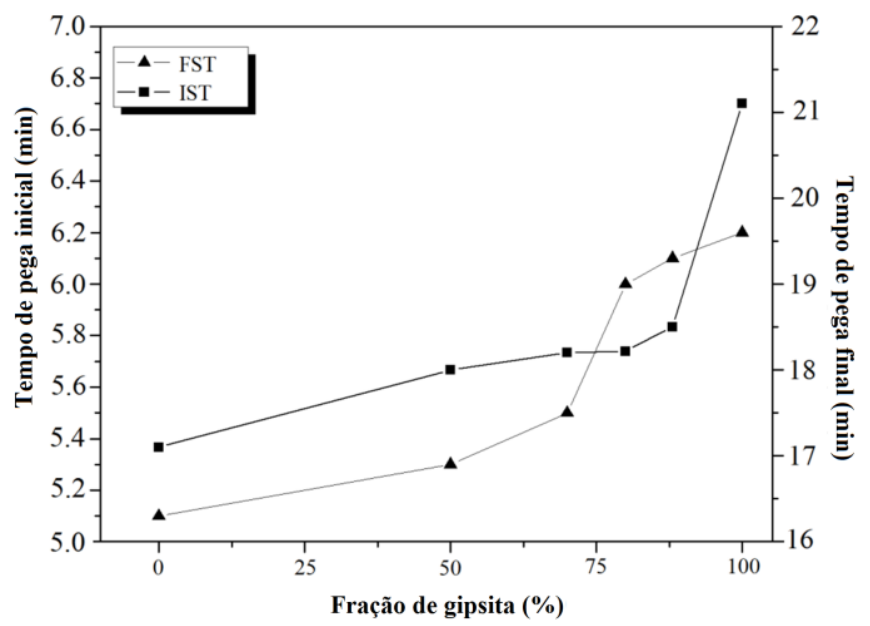

Figura 6 - Tempos de pega inicial e final do reciclado de gesso beta (resíduos de bloco de gesso, $\mathrm{Tc}=500^{\circ} \mathrm{C}, \mathrm{Qs}=175 \mathrm{~kg} / \mathrm{h}$ e $\left.\mathrm{n}=2 \mathrm{rpm}\right)$

No entanto, os resultados para o tempo do ajuste final não cumprem os requisitos legais brasileiros, exceto quando calcinação é realizada com $100 \%$ de minério de gesso. A produção de um emplastro beta reciclado com tempos iniciais e finais de configuração adequada é possível, mas requer um hemi-hidrato de sulfato de cálcio gerado no primeiro ciclo, com melhores propriedades físicas e mecânicas.

\section{CONCLUSÕES}

Os resultados do presente estudo demonstram a otimização de um processo de calcinação de gesso resíduos em um forno rotativo através de um planejamento fatorial completo com pontos centrais para se obter um modelo matemático quadrático -linear que combina os fatores e suas interações. As superfícies curvas de contorno e de resposta demonstram condições ideais de operação em relação ao grau de hidratação em função da temperatura de combustão, o fluxo de alimentação de sólidos e a velocidade de rotação. $O$ tratamento dos RCD através do processo de calcinação optimizado produzido um hemi-hidrato de sulfato de cálcio com propriedades físicas e mecânicas em conformidade com as especificações exigidas pelas normas brasileiras. Um índice de reciclagem de 
$20 \%$ foi alcançado, o que é equivalente a uma fração maior de gesso calcinado resíduos capazes de produzir um gesso muito perto dos requisitos estabelecidos pelas normas brasileiras. Por isso, é possível reutilizar e comercializar os resíduos de gesso gerados pela indústria da construção civil, após o tratamento adequado. Isto representa uma alternativa promissora para este tipo de resíduos, evitando o descarte do material em aterros sanitários.

\section{REFERÊNCIAS}

Associação Brasileira de Normas Técnicas - ABNT. Gesso para construção civil: determinação de propriedades físicas do pó: método de ensaio - NBR/12127. Rio de Janeiro, 1991, 4p.

Associação Brasileira de Normas Técnicas - ABNT. Gesso para construção civil: determinação de propriedades mecânicas: método de ensaio - NBR/12129. Rio de Janeiro, 1991, 5p.

BALTAR, C. A. M.; BASTOS, F. F. e LUZ, A. B. Diagnóstico do poço gesseiro de Pernambuco (brasil) com ênfase na produção de gipsita para a fabricação de cimento. In: IV Jornadas Iberoamericanas de Materiales de Construcción, Anais. Tegucigalpa, Honduras, 2004.

BALTAR, C. A. M.; BASTOS, F. F. e LUZ, A. B. Rochas \& minerais industriais: gipsita. 2.ed. Rio de Janeiro: CETEM/MCT, 989 p. 2008.

LAMBE, C.M., OFFUTT, J.S., Consistency classification of industrial plasters. Ceramic Bulletin. 33 (1954) 272-276.

SILVA, J. B.;DUTRA, R. P. S.; NASCIMENTO, R, M; MARTINELLI, A.E.; GOMES, U. U. Avaliação da incorporação de rejeitos de gesso de construção em formulações de cerâmica. Congresso Brasileiro de Engenharia e Ciência dos Materiais (CBECIMat) Foz do Iguaçu, V.17, p.2149-2160, novembro de 2006.

SINDUSCON-MG. Cartilha de gerenciamento de resíduos sólidos para a construção civil. Superintendência da Prefeitura de Belo Horizonte. Emitido em 2005. Disponível em: http://www.cepam.sp.gov.br/arquivos/sisnama/meio_ambiente_em_temas/sindusco n2_ma.pdf. Acesso em 20 de julho de 2010.

SINGH, N.B., MIDDENDORF, B.; Calcium sulphate hemihydrate hydration leading to gypsum crystallization, Prog. Cryst. Growth Charact. Mater. 53 (2007) 57-77. 